\title{
Global linear gyrokinetic particle-in-cell simulations including electromagnetic effects in shaped plasmas
}

\author{
A. Mishchenko*, M. Borchardt, M. Cole, R. Kleiber, A. Könies, and A. Zocco \\ Max Planck Institute for Plasma Physics, \\ Wendelsteinstr. 1, 17491 Greifswald, Germany \\ R. Hatzky and T. Fehér \\ Max Planck Institute for Plasma Physics, \\ Boltzmannstr. 2, 85748 Garching, Germany
}

(Dated: April 2, 2015)

\begin{abstract}
We give an overview of recent developments in electromagnetic simulations based on the gyrokinetic particle-in-cell codes GYGLES and EUTERPE. We present the gyrokinetic electromagnetic models implemented in the codes and discuss further improvements of the numerical algorithm, in particular the so-called pullback mitigation of the cancellation problem. The improved algorithm is employed to simulate linear electromagnetic instabilities in shaped tokamak and stellarator plasmas, which was previously impossible for the parameters considered.
\end{abstract}

*alexey.mishchenko@ipp.mpg.de 


\section{INTRODUCTION}

Electromagnetic kinetic effects are of great importance in fusion plasmas for a wide variety of phenomena. These include, for instance, Alfvénic dynamics, tearing modes, and electromagnetic drift micro-instabilities. Since all these phenomena have characteristic frequencies lower than the gyro-frequency, one can use the gyrokinetic approach, resulting in a substantial reduction in computational costs. However, gyrokinetic electromagnetic simulations suffer from the cancellation problem $[1,2]$. This problem has been addressed within the particle-in-cell (PIC) framework [2-5] as well as the Eulerian approach [6]. It has been possible to perform global fully-gyrokinetic simulations of a large class of Alfvénic modes, including their interaction with the fast particles. An overview of this work will be given below. Despite substantial progress, a complete solution of the cancellation problem has remained elusive, in particular for global modes in realistically shaped toroidal devices. Recently, a new approach, based on mixed coordinates [7] and pullback mitigation [8], has been suggested. This approach has the potential to mitigate the cancellation problem considerably. In this paper, we implement pullback mitigation to globally simulate shaped plasmas of an elongated tokamak and the Wendelstein 7-X [9] stellarator (W7-X). The fully gyrokinetic system of equations is solved in these simulations for all particle species.

We give an overview of recent developments in electromagnetic simulations based on the gyrokinetic PIC codes GYGLES and EUTERPE. The code GYGLES is a linear global (full radius, full flux surface) gyrokinetic PIC code that is able to simulate up to three kinetic species (ions, electrons and fast ions) in axisymmetric equilibria. It solves the field equations for the electrostatic and parallel vector potentials. The most advanced fully-gyrokinetic electromagnetic PIC simulations in tokamak and pinch geometries reported so far [10-14] have been performed with the GYGLES code. The code EUTERPE is an extension of the GYGLES code to permit nonlinear simulations and non-axisymmetric (VMEC [15]) equilibria.

Both codes have been applied to simulate Alfvénic instabilities in fusion-relevant plasmas. Global Alfvén Eigenmodes [10], Toroidal Alfvén Eigenmodes (TAE) [10], their interaction with fast particles [11] and the Alfvénic continuum [12, 14], and modification of TAE instabilities into Energetic Particle Modes (EPM) [11, 14] have all been successfully studied with the GYGLES code. Recently, the gyrokinetic internal $m=1$ kink mode and $m=1$ 
reconnecting mode have been simulated [13] with GYGLES in screw pinch geometry.

The global gyrokinetic particle-in-cell code EUTERPE contains a hierarchical collection of numerical tools of increasing complexity including a simple perturbative scheme (CKAEUTERPE, see Refs. [16, 17]), based on the reduced-MHD eigenvalue solver CKA [18]; a more advanced hybrid self-consistent fluid-electron gyrokinetic-ion model [19]; and the most comprehensive, but also most computationally expensive, fully gyrokinetic model. The cancellation problem $[1,2]$, being the major obstacle for full-gyrokinetic simulations, does not appear in the reduced models, which therefore become flexible tools suitable for approximate studies of certain parameter regimes. Such a study has been undertaken [17] in stellarator geometry (Wendelstein 7-X and HELIAS reactor) using the perturbative CKAEUTERPE tool. Interesting regions of the parametric space, unstable with respect to Alfvén Eigenmodes, have been identified. This information is a robust starting point for further work with the more advanced physical models. The fluid-electron gyrokinetic-ion hybrid model has been used extensively [19] to simulate internal kink modes and fishbones in tokamak geometries, based on numerical equilibria at realistic aspect ratio and a finite elongation. The fully-gyrokinetic electromagnetic model of the EUTERPE code has been verified using the International Tokamak Physics Activity (ITPA) benchmark [20]. Further improvements of the cancellation scheme [2-5] have been considered and presented in Refs. [7,

8]. These schemes are required to provide numerical stability when simulating electron kinetics in electromagnetic regimes using the fully gyrokinetic description.

The structure of this paper is as follows. In Sec. II, the entire GYGLES/EUTERPE toolset is presented for completeness. The standard gyrokinetic formulation is shortly described in Sec. II A; the mixed-variable formulation is discussed in Sec. II B; the reduced models are addressed in Sec. III A and in Sec. III B. We describe our simulations in shaped tokamak and stellarator (W7-X) plasmas in Sec. IV and conclude in Sec. V.

\section{GYROKINETIC MODEL}

\section{A. Standard formulation}

The most comprehensive model available in the GYGLES and EUTERPE codes is the gyrokinetic Vlasov-Maxwell system of equations [21]. Each distribution function is split 
into a background part and a perturbation $f_{s}=F_{0 s}+\delta f_{s}$ with $s=\mathrm{i}$, e, f indicating the particle species (bulk plasma ions and electrons, fast particles). The background distribution function is taken to be a Maxwellian. The perturbed distribution function $\delta f_{s}$ is found from the linearized gyrokinetic Vlasov equation:

$$
\frac{\partial \delta f_{s}}{\partial t}+\dot{\mathbf{R}}^{(0)} \cdot \frac{\partial \delta f_{s}}{\partial \mathbf{R}}+\dot{v}_{\|}^{(0)} \frac{\partial \delta f_{s}}{\partial v_{\|}}=-\dot{\mathbf{R}}^{(1)} \cdot \frac{\partial F_{0 s}}{\partial \mathbf{R}}-\dot{v}_{\|}^{(1)} \frac{\partial F_{0 s}}{\partial v_{\|}}
$$

Here, $\left[\dot{\mathbf{R}}^{(0)}, \dot{v}_{\|}^{(0)}\right]$ correspond to the unperturbed gyrocenter trajectories, while $\left[\dot{\mathbf{R}}^{(1)}, \dot{v}_{\|}^{(1)}\right]$ are the perturbations of the trajectories (proportional to the electromagnetic field fluctuations). Note that $v_{\|}$is not equal to the physical parallel velocity in this notation. Employing the Hamiltonian formulation [21], the equations of motion are

$$
\begin{aligned}
\dot{\mathbf{R}} & =\left(v_{\|}-\frac{q}{m}\left\langle A_{\|}\right\rangle\right) \mathbf{b}^{*}+\frac{1}{q B_{\|}^{*}} \mathbf{b} \times\left[\mu \nabla B+q\left(\nabla\langle\phi\rangle-v_{\|} \nabla\left\langle A_{\|}\right\rangle\right)\right] \\
\dot{v}_{\|} & =-\frac{1}{m}\left[\mu \nabla B+q\left(\nabla\langle\phi\rangle-v_{\|} \nabla\left\langle A_{\|}\right\rangle\right)\right] \cdot \mathbf{b}^{*}
\end{aligned}
$$

Here, $\phi$ and $A_{\|}$are the perturbed electrostatic and magnetic potentials, $\mu$ is the magnetic moment, $m$ is the mass of the particle, $B_{\|}^{*}=\mathbf{b} \cdot \nabla \times \mathbf{A}^{*}, \mathbf{b}^{*}=\nabla \times \mathbf{A}^{*} / B_{\|}^{*}, \mathbf{A}^{*}=\mathbf{A}_{0}+$ $\left(m v_{\|} / q\right) \mathbf{b}$ is the modified vector potential, $\mathbf{A}_{0}$ is the magnetic potential corresponding to the equilibrium magnetic field, $\mathbf{B}=\nabla \times \mathbf{A}_{0}, \mathbf{b}=\mathbf{B} / B$ is the unit vector in the direction of the equilibrium magnetic field, and the gyro-averaged potentials are defined as usual:

$$
\langle\phi\rangle=\oint \frac{\mathrm{d} \theta}{2 \pi} \phi(\mathbf{R}+\boldsymbol{\rho}),\left\langle A_{\|}\right\rangle=\oint \frac{\mathrm{d} \theta}{2 \pi} A_{\|}(\mathbf{R}+\boldsymbol{\rho})
$$

with $\boldsymbol{\rho}$ the gyroradius of the particle and $\theta$ the gyro-phase. The perturbed electrostatic and magnetic potentials are found self-consistently from the gyrokinetic quasineutrality equation and parallel Ampère's law (see Ref. [5] for numerical details):

$$
-\nabla \cdot\left[\left(\sum_{s=\mathrm{i}, \mathrm{f}} \frac{q_{s}^{2} n_{s}}{T_{s}} \rho_{s}^{2}\right) \nabla_{\perp} \phi\right]=\sum_{s=\mathrm{i}, \mathrm{e}, \mathrm{f}} q_{s} n_{1 s}, \quad\left(\sum_{s=\mathrm{i}, \mathrm{e}, \mathrm{f}} \frac{\hat{\beta}_{s}}{\rho_{s}^{2}}-\nabla_{\perp}^{2}\right) A_{\|}=\mu_{0} \sum_{s=\mathrm{i}, \mathrm{e}, \mathrm{f}} j_{\| 1 s},
$$

where $n_{1 s}=\int \mathrm{d}^{6} Z \delta f_{s} \delta(\mathbf{R}+\boldsymbol{\rho}-\mathbf{x})$ is the perturbed gyrocenter density, $j_{\| 1 s}=q_{s} \int \mathrm{d}^{6} Z \delta f_{s} v_{\|} \delta(\mathbf{R}+\boldsymbol{\rho}-\mathbf{x})$ is the perturbed gyrocenter current, $q_{s}$ is the charge of the particle, $\mathrm{d}^{6} Z=B_{\|}^{*} \mathrm{~d} \mathbf{R} \mathrm{d} v_{\|} \mathrm{d} \mu \mathrm{d} \theta$ is the phase-space volume, $\rho_{s}=\sqrt{m_{s} T_{s}} /(e B)$ is the thermal gyroradius and $\hat{\beta}_{s}=\mu_{0} n_{0 s} T_{s} / B^{2}$ is half the plasma species beta. The polarization density is treated in the long-wavelength approximation and finite Larmor radius (FLR) effects are neglected for electrons. The zeroth-order densities of the particle species satisfy 
the quasineutrality equation $\sum_{s} q_{s} n_{0 s}=0$ with $s=\mathrm{i}$, e, f. In EUTERPE, the nonlinear and collisional versions of the gyrokinetic system of equations are available, whereas GYGLES is a linear axisymmetric collisionless code.

\section{B. Mixed-variable formulation}

In the mixed-variable formulation $[7,8]$ of the gyrokinetic theory, we deliberately split the magnetic potential into 'symplectic' and 'Hamiltonian' parts:

$$
A_{\|}=A_{\|}^{(\mathrm{s})}+A_{\|}^{(\mathrm{h})}
$$

This naming is inspired by Ref. [21]; the precise relation will become more clear in the following. In these notations, the perturbed guiding-center phase-space Lagrangian [21] is

$$
\gamma=q \mathbf{A}^{*} \cdot \mathrm{d} \mathbf{R}+\frac{m}{q} \mu \mathrm{d} \theta+q A_{\|}^{(\mathrm{s})} \mathbf{b} \cdot \mathrm{d} \mathbf{x}+q A_{\|}^{(\mathrm{h})} \mathbf{b} \cdot \mathrm{d} \mathbf{x}-\left[\frac{m v_{\|}^{2}}{2}+\mu B+q \phi\right] \mathrm{d} t
$$

We now perform the Lie transform [21] in such a way that the 'Hamiltonian part' $A_{\|}^{(\mathrm{h})}$ contributes to the gyrokinetic Hamiltonian, whereas the 'symplectic part' $A_{\|}^{(\mathrm{s})}$ enters the gyrokinetic symplectic structure. The resulting gyrokinetic phase-space Lagrangian is written to first order:

$$
\Gamma=q \mathbf{A}^{*} \cdot \mathrm{d} \mathbf{R}+\frac{m}{q} \mu \mathrm{d} \theta+q\left\langle A_{\|}^{(\mathrm{s})}\right\rangle \mathbf{b} \cdot \mathrm{d} \mathbf{R}-\left[\frac{m v_{\|}^{2}}{2}+\mu B+q\left\langle\phi-v_{\|} A_{\|}^{(\mathrm{h})}\right\rangle\right] \mathrm{d} t
$$

The formulation Eq. (8) is neither Hamiltonian nor symplectic and will, therefore, be dubbed the 'mixed-variable' formulation, following Ref. [7]. The corresponding perturbed equations of motion are

$$
\begin{aligned}
& \dot{\mathbf{R}}^{(1)}=\frac{\mathbf{b}}{B_{\|}^{*}} \times \nabla\left\langle\phi-v_{\|} A_{\|}^{(\mathrm{s})}-v_{\|} A_{\|}^{(\mathrm{h})}\right\rangle-\frac{q}{m}\left\langle A_{\|}^{(\mathrm{h})}\right\rangle \mathbf{b}^{*} \\
& \dot{v}_{\|}^{(1)}=-\frac{q}{m}\left[\mathbf{b}^{*} \cdot \nabla\left\langle\phi-v_{\|} A_{\|}^{(\mathrm{h})}\right\rangle+\frac{\partial}{\partial t}\left\langle A_{\|}^{(\mathrm{s})}\right\rangle\right]-\frac{\mu}{m} \frac{\mathbf{b} \times \nabla B}{B_{\|}^{*}} \cdot \nabla\left\langle A_{\|}^{(\mathrm{s})}\right\rangle
\end{aligned}
$$

For the scheme to work, an equation for $\partial A_{\|}^{(\mathrm{s})} / \partial t$ is needed. We find useful to employ

$$
\frac{\partial}{\partial t} A_{\|}^{(\mathrm{s})}+\mathbf{b} \cdot \nabla \phi=0
$$

The zeroth-order gyrocenter characteristics remain unchanged. The perturbed mixedvariable distribution function is found from the gyrokinetic Vlasov equation. The electrostatic potential and the 'Hamiltonian part' of the magnetic potential are found, respectively, from the gyrokinetic quasineutrality equation and mixed-variable parallel Ampère's 
law, which takes now the form:

$$
\left(\sum_{s=\mathrm{i}, \mathrm{e}, \mathrm{f}} \frac{\hat{\beta}_{s}}{\rho_{s}^{2}}-\nabla_{\perp}^{2}\right) A_{\|}^{(\mathrm{h})}=\mu_{0} \sum_{s=\mathrm{i}, \mathrm{e}, \mathrm{f}} j_{\| 1 s}+\nabla_{\perp}^{2} A_{\|}^{(\mathrm{s})}
$$

with the usual notation. Invoking the pullback transformation [21], one can express the distribution function in the symplectic formulation in terms of the mixed-variable distribution function as follows:

$$
f_{1 s}^{(\mathrm{s})}=f_{1 s}^{(\mathrm{m})}+\frac{q_{s}\left\langle A_{\|}^{(\mathrm{h})}\right\rangle}{m_{s}} \frac{\partial F_{0 s}}{\partial v_{\|}}
$$

We proceed modifying the standard algorithm [5] as follows.

1. At the end of each time step, we redefine the magnetic potential splitting, Eq. (6), such that the entire instantaneous value of the parallel magnetic potential $A_{\|}\left(t_{i}\right)$ is collected in its 'symplectic part':

$$
A_{\|(\text {new })}^{(\mathrm{s})}\left(t_{i}\right)=A_{\|}\left(t_{i}\right)=A_{\|(\text {old })}^{(\mathrm{s})}\left(t_{i}\right)+A_{\|(\text {old })}^{(\mathrm{h})}\left(t_{i}\right)
$$

2. As a consequence of the new splitting, Eq. (14), the 'Hamiltonian' part of the vector potential must be corrected to preserve the total $A_{\|}$:

$$
A_{\|(\text {new })}^{(\mathrm{h})}\left(t_{i}\right)=0
$$

3. For this modified splitting, the new mixed-variable distribution function must coincide with its symplectic formulation counterpart. The symplectic formulation distribution function can be found invoking the pullback, Eq. (13), using the old values of the mixed-variable distribution function and the 'Hamiltonian' part of the parallel vector potential:

$$
f_{1 s \text { (new) }}^{(\mathrm{m})}\left(t_{i}\right)=f_{1 s}^{(\mathrm{s})}\left(t_{i}\right)=f_{1 s(\mathrm{old})}^{(\mathrm{m})}\left(t_{i}\right)+\frac{q_{s}\left\langle A_{\| \text {(old) }}^{(\mathrm{h})}\left(t_{i}\right)\right\rangle}{m_{s}} \frac{\partial F_{0 s}}{\partial v_{\|}}
$$

The values of $f_{1 s(\mathrm{old})}^{(\mathrm{m})}$ and $A_{\|(\mathrm{old})}^{(\mathrm{h})}$ are found solving, respectively, the gyrokinetic equation and Ampère's law, Eq. (12), at the current time step $t_{i}$.

4. Proceed, explicitly solving the mixed-variable system of equations (9)-(12) at the next time step $t_{i}+\Delta t$ in the usual way, but using Eqs. (14)-(16) as the initial conditions.

This rearrangement between the symplectic and the Hamiltonian components of the 'initial conditions' has to be done at each time step. Note that in general the parallel dynamics 
is determined by the time derivative of the total magnetic potential $\partial A_{\|} / \partial t$, whereas the terms responsible for the cancellation problem are proportional to its instantaneous value $A_{\|}(t)$. In our approach, we force the 'symplectic part' $A_{\|}^{(\mathrm{s})}$ to be a dominant contribution to this value. The small residual $A_{\|}^{(\mathrm{h})}$ is self-consistently computed at each time step from the gyrokinetic system of equations in the mixed-variable formulation, thus guaranteeing correctness of the physical quantity $\partial A_{\|} / \partial t$, in accordance with the actual dynamics of the system. This scheme is not limited to Alfvénic systems, which obey $E_{\|} \approx 0$. It supplements prior developments [5] to treat the cancellation problem and can reduce considerably the numerical effort required (see Ref. [8] for details). In nonlinear case, the scheme can also be used at the cost of the parallel nonlinearity being neglected. The truncation is related to the implicit linearisation during the pullback step, Eq. (16). Further generalisations of the scheme will be considered and reported elsewhere.

\section{REDUCED MODELS}

\section{A. Fluid-electron model}

The gyrokinetic model, although being comprehensive, can require a substantial numerical effort. In addition, it is sometimes desirable to intentionally admit or exclude certain physical effects such as the effect of the electron pressure gradient in a flexible way, which may be impossible within the gyrokinetic formulation. We have therefore developed a hybrid model which evolves the ion and fast ion distribution functions using the gyrokinetic PIC method employing the symplectic formulation [21], while electron effects are treated by the evolution of fluid moments. The electron continuity equation is

$$
\begin{aligned}
\frac{\partial n_{1 \mathrm{e}}}{\partial t}+n_{0} \mathbf{B} & \cdot \nabla\left(\frac{u_{\| 1 \mathrm{e}}}{B}\right)+B \mathbf{v}_{\mathrm{E}} \cdot \nabla\left(\frac{n_{0}}{B}\right)+\left(\nabla \times A_{\|} \mathbf{b}\right) \cdot \nabla\left(\frac{n_{0} u_{\| 0 \mathrm{e}}}{B}\right) \\
+ & n_{0}\left(\delta \mathbf{v}_{*}-\mathbf{v}_{E}\right) \cdot \frac{\nabla B}{B}+\frac{\nabla \times \mathbf{B}}{B^{2}} \cdot\left[-\frac{\nabla p_{1 \mathrm{e}}}{e}+n_{0} \nabla \phi\right]=0
\end{aligned}
$$

where

$$
\mathbf{v}_{\mathrm{E}}=\frac{\mathbf{b} \times \nabla \phi}{B}, \quad \delta \mathbf{v}_{*}=2 \frac{\mathbf{b} \times \nabla p_{1 \mathrm{e}}}{n_{0} m_{\mathrm{e}} e B}
$$

The equation for the perturbed pressure is

$$
\frac{\partial p_{1 \mathrm{e}}}{\partial t}=-\mathbf{v}_{\mathrm{E}} \cdot \nabla p_{0}=-\frac{\mathbf{b} \times \nabla \phi}{B} \cdot \nabla n_{0} T_{0}
$$


The magnetic potential is found solving the ideal Ohm's law:

$$
E_{\|}=-\nabla_{\|} \phi-\frac{\partial A_{\|}}{\partial t}=0
$$

and the parallel electron velocity is found from Ampère's law:

$$
e n_{0} u_{\| 1 \mathrm{e}}=\sum_{s=\mathrm{i}, \mathrm{f}} j_{\| 1 s}+\frac{1}{\mu_{0}} \nabla_{\perp}^{2} A_{\|}
$$

The electrostatic potential is obtained from the usual gyrokinetic quasineutrality equation, see Eq. (5).

Where kinetic bulk ion contributions are neglected, the fluid equations simplify to reduced ideal MHD. Taking the sum $q_{\mathrm{i}} \partial n_{1 \mathrm{i}} / \partial t+q_{\mathrm{e}} \partial n_{1 \mathrm{e}} / \partial t$ and using equation (17) with species labels $\mathrm{i}$ and e respectively, we arrive at an equivalent equation in terms of the charge and parallel current densities. It is possible in this way to treat both electrons and bulk ions as a single fluid, while modelling fast ions gyrokinetically.

\section{B. Perturbative hybrid MHD model}

In the computationally most robust and simple perturbative hybrid MHD approach, one finds the perturbed electromagnetic fields (the radial structure and the frequency) by solving the reduced ideal-MHD vorticity equation:

$$
\begin{array}{r}
\omega^{2} \nabla \cdot\left(\frac{1}{v_{\mathrm{A}}^{2}} \nabla_{\perp} \phi\right)+\nabla \cdot\left[\mathbf{b} \nabla_{\perp}^{2}(\mathbf{b} \cdot \nabla) \phi\right]-\nabla \cdot\left(\frac{\mu_{0} j_{\| 0}}{B}[\nabla \times(\boldsymbol{b}(\boldsymbol{b} \cdot \nabla \phi))]_{\perp}\right)- \\
-\nabla \cdot\left(\frac{2 \mu_{0}}{B^{2}}\left[(\mathbf{b} \times \nabla \phi) \cdot \nabla p_{0}\right](\mathbf{b} \times \boldsymbol{\kappa})\right)=0
\end{array}
$$

Here, $v_{\mathrm{A}}$ is the Alfvén velocity, $j_{\| 0}$ is the ambient parallel current, $p_{0}$ is the background plasma pressure, and $\boldsymbol{\kappa}=(\mathbf{b} \cdot \nabla) \mathbf{b}$ is the magnetic field-line curvature. The perturbed parallel magnetic potential $A_{\|}$is found from the ideal Ohm's law, Eq. (20). The fast ions are treated solving the linear or nonlinear gyrokinetic equation including all finite orbit width and finite gyroradius effects. 


\section{SIMULATIONS}

\section{A. General remarks}

The schemes described above are discretised equivalently in EUTERPE and GYGLES, the perturbation of the distribution functions being represented with markers and the field quantities with finite elements. In the fluid-electron model, the fluid moments are also discretised with finite elements. Many physical phenomena have been considered with these codes (see Sec. I for an overview). All the models available in the GYGLES/EUTERPE toolset have been cross-benchmarked and verified within the framework of the International Tokamak Physics Activity [20].

In this paper, gyrokinetic mixed-variable and reduced fluid-electron simulations using the EUTERPE code in shaped tokamak and stellarator (W7-X) plasmas will be considered.

\section{B. Linear electromagnetic simulations in elongated tokamak geometry}

First, we consider a TAE in a tokamak configuration $[11,20]$ with the minor radius $r_{\mathrm{a}}=1 \mathrm{~m}$, the major radius $R_{0}=10 \mathrm{~m}$, the magnetic field on axis $B_{0}=3 \mathrm{~T}$, and the safety factor profile $q(r)=1.71+0.16\left(r / r_{\mathrm{a}}\right)^{2}$ (here, $r$ is the small radius). The background plasma profiles (corresponding to Maxwellian unperturbed distribution functions) are chosen to be flat with the ion (hydrogen) density $n_{\mathrm{i}}=2 \times 10^{19} \mathrm{~m}^{-3}$, the electron density defined by the quasi-neutrality $n_{\mathrm{e}}=n_{\mathrm{i}}+n_{\mathrm{f}}$, and flat bulk-species temperatures $T_{\mathrm{i}}=T_{\mathrm{e}}=1 \mathrm{keV}$. A Maxwellian is also chosen for the unperturbed distribution function of the fast particles (deuterium ions). The fast-particle temperature $T_{\mathrm{f}}=0.4 \mathrm{MeV}$ is also flat and the fastparticle density is given by the expression:

$$
n_{\mathrm{f}}\left(s_{\mathrm{pol}}\right)=n_{\mathrm{of}} \exp \left[-\frac{\Delta_{\mathrm{nf}}}{L_{\mathrm{nf}}} \tanh \left(\frac{s_{\mathrm{pol}}-s_{\mathrm{nf}}}{\Delta_{\mathrm{nf}}}\right)\right]
$$

with $s_{\text {pol }}$ being the square root of the normalised poloidal flux, $s_{\text {nf }}=0.5$ the position of the maximal value of $\kappa_{\mathrm{nf}}=\left|\nabla n_{\mathrm{f}}\right| / n_{\mathrm{f}}, n_{0 \mathrm{f}}=0.75 \times 10^{17} \mathrm{~m}^{-3}$ the fast particle density at $s_{\mathrm{pol}}=s_{\mathrm{nf}}, \Delta_{\mathrm{nf}}=0.2$ the characteristic width of the density profile, and $L_{\mathrm{nf}}=0.3$ determining the strength of the fast particle density gradient. We note here that the Maxwellian distribution function is the simplest choice sufficient for the proof-of-principles simulations such as described in this paper. Since we use the $\delta f$ approach, $F_{0}$ is fixed and does not 
change during the simulations. Hence, such issues as a relaxation of $F_{0}$ to the nearby equilibrium do not appear in praxis. It is, therefore, safe to use such a choice of the distribution function also within the mixed-variable formalism. We agree, however, that a more realistic distribution function must be implemented in future studies, especially for fast ions when such heating methods, such as the Neutral Beam Injection, are considered.

In the configuration described, we simulate the TAE with the toroidal mode number $n=-6$ and the dominant poloidal harmonics $m=10$ and $m=11$. The ambient magnetic field is computed numerically using the VMEC code [15]. In this code, the magnetic field is determined by the safety factor; the ambient plasma pressure profile, neglected for lowbeta magnetic configurations considered here; and the shape of the plasma boundary whose cylindrical coordinates $\left[R_{\mathrm{c}}(\theta), Z_{\mathrm{c}}(\theta)\right]$ are given by the expressions:

$$
R_{\mathrm{c}}(\theta)=R_{0}+r_{\mathrm{a}} \cos \theta, \quad Z_{\mathrm{c}}(\theta)=\kappa r_{\mathrm{a}} \sin \theta
$$

Here, $\theta$ is the poloidal angle and $\kappa$ is the elongation of the plasma cross-section. In the following we will vary the elongation from $\kappa=1.0$ (circular cross-section) to $\kappa=1.8$ (elongated tokamak). It has been observed in previous simulations that the cancellation problem becomes more severe at a finite elongation. It was hypothesised that this is caused by an enhanced poloidal mode coupling in shaped plasmas. This coupling appears to amplify certain inconsistencies in the cancellation terms related to the side band formation which are hard to capture by the standard cancellation scheme [2-5]. In future, a more detailed and rigorous understanding of this issue can be developed. In the present publication, we circumvent this problem using mixed variables [7] and the pullback mitigation scheme [8].

In Fig. 1, the frequency of the TAE is shown as a function of the elongation. The modification of the frequency is related to the deformation of the shear Alfvén continuum by the elongation, which creates new gaps in the continuum structure. To show this deformation, we compare the shear Alfvén continua at $\kappa=1$, in Fig. 2, and at $\kappa=1.8$, in Fig. 3. In Fig. 2 (circular cross-sections), one sees only the toroidicity gap, with the TAE frequency indicated by the red dashed line. In contrast, in Fig. 3 (elongated tokamak), both the toroidicity and the ellipticity gaps appear in the spectrum. The toroidal gap together with the corresponding TAE frequency (indicated with the red dashed line) are shifted to lower frequencies by the elongation, which introduces new couplings of the poloidal Fourier harmonics and therefore creates additional gaps in the continuum. The growth rate of the 
TAE decreases with the elongation, as shown in Fig. 4. Note that a similar finding has been reported in Ref. [22]. In Figs. 1 and 4, we compare our fully-gyrokinetic simulations with the corresponding fluid-electron simulations and find a good agreement. In Fig. 5, we show the eigenmode structure of the electrostatic potential, here computed at the elongation $\kappa=1.2$. A typical TAE structure can be seen at this elongation. A similar mode has also been observed at $\kappa=1.8$ shown in Fig. 6 . Here, one sees that the coupling to the poloidal sidebands is stronger compared to the previous case.

\section{Linear electromagnetic simulations in Wendelstein 7-X geometry}

Now, we consider the Wendelstein 7-X [9] stellarator. This machine is the world-wide largest optimised stellarator and will begin its operation soon. Here, we present global gyrokinetic simulations of Ion-Temperature-Gradient (ITG) driven modes computed including electromagnetic effects.

In our simulations, we distinguish between the 'equilibrium beta' $\beta_{\text {eq }}$ used to compute the ambient magnetic field and the 'gyrokinetic beta' determining the bulk plasma profiles. The 'equilibrium beta' determines the degree of the stellarator optimisation whereas the 'gyrokinetic beta' is responsible for the Alfvénic dynamics in the given ambient magnetic field. In this paper, we choose the ambient magnetic field, computed numerically using the VMEC code [15], in such a way that $\left\langle\beta_{\text {eq }}\right\rangle=2 \mu_{0}\langle p\rangle /\left\langle B^{2}\right\rangle=3 \%$ with the pressure profile given by the expression $p / p(0)=1-1.67 s+0.67 s^{2}$. Here, $\langle\ldots\rangle$ denotes the volume average and $s$ is the normalised toroidal flux.

The bulk plasma profiles are chosen to have $\beta_{*}=\mu_{0} n_{*} T_{*} / B_{*}^{2}=0.005$ corresponding to 'gyrokinetic' $\beta=4 \beta_{*}=2 \%$. Here $n_{*}$ is the plasma density averaged over the entire plasma volume, $T_{*}=T_{\mathrm{e}}(s=0.5)$ is the characteristic electron temperature (with $s$ being the normalised toroidal flux) and $B_{*}=B(s=0, \zeta=0)$ is the characteristic ambient magnetic field (with $\zeta$ being the toroidal angle). The plasma size is determined by the parameter $L_{x}=458.2$ which is approximately the ratio $L_{x} \approx 2.2 r_{\mathrm{a}} / \rho_{\mathrm{s}}$ with $r_{\mathrm{a}}$ being the average minor radius of the non-axisymmetric device and $\rho_{\mathrm{s}}=\sqrt{m_{\mathrm{i}} T_{*}} /\left(e B_{*}\right)$ the characteristic ion sound gyroradius. The plasma density and temperature profiles are defined as functions of the 
normalised toroidal flux according to the expressions:

$$
\begin{aligned}
& n_{(\mathrm{i}, \mathrm{e})}(s)=n_{0} \exp \left[-\frac{\Delta_{\mathrm{n}}}{L_{\mathrm{n}}} \tanh \left(\frac{s-s_{0}}{\Delta_{\mathrm{n}}}\right)\right] \\
& T_{(\mathrm{i}, \mathrm{e})}(s)=T_{0} \exp \left[-\frac{\Delta_{\mathrm{T}(\mathrm{i}, \mathrm{e})}}{L_{\mathrm{T}(\mathrm{i}, \mathrm{e})}} \tanh \left(\frac{s-s_{0}}{\Delta_{\mathrm{T}(\mathrm{i}, \mathrm{e})}}\right)\right]
\end{aligned}
$$

with $s_{0}=0.5, \Delta_{\mathrm{n}}=0.3,1 / L_{\mathrm{Ti}}=3.5, \Delta_{\mathrm{Ti}}=0.3$, and a flat electron temperature profile. The parameters $n_{0}$ and $T_{0}$ are determined by the plasma size $L_{x}$ and its pressure $\beta_{*}$. For this choice, the volume-averaged 'equilibrium beta' is approximately the volume-averaged 'gyrokinetic beta' although the profiles differ. In our simulations, we prefer to change the plasma profiles while keeping the ambient magnetic configuration fixed. This procedure allows to separate effects of the plasma profiles on the mode stability from those of the ambient magnetic field. In future, role of the finite-beta modifications of the magnetic equilibrium will be addressed, too. Here, we remark only that the particle orbits in the vacuum and in a high-beta W7-X stellarator configurations may differ substantially.

First, we consider the case with a flat density profile. The dominant poloidal and toroidal mode numbers are, respectively, $m_{0}=58$ and $n_{0}=-47$. The poloidal mode number for the parameters chosen $k_{\theta} \rho_{\mathrm{s}} \approx 2.2 m_{0} / L_{x}=0.279$. In this case, there is a global unstable mode, whose temporal evolution is shown in Fig. 7. One sees that the mode evolution is approximately harmonic. The long-time quasi-mode transient effects may also be present but their detailed evaluation is beyond the scope of our simulations. The poloidal mode decomposition of $\phi(s, \theta, \zeta=0)$ is shown in Fig. 8. One sees that the mode shows some slab-like features, such as a dominant harmonic. Note that the slab-like character of the mode is expected in $\mathrm{W} 7-\mathrm{X}$ even for $k_{\theta} \rho_{\mathrm{s}} \approx 0.3$, in contrast to tokamaks, since the connection length between bad and good curvature is much smaller [23] in W7-X than in an equivalent tokamak. A similar observation can be made in Fig. 9, where the Fourier spectrum of the electrostatic potential measured at $s=0.5$ is shown. This spectrum has a well-pronounced dominant Fourier harmonic, as is expected for slab-like modes.

In Fig. 10, the poloidal cross-section of the electrostatic potential is shown at the toroidal angle $\zeta=0$. One can see mode activity both at the inner and the outer side of the torus, again in contrast to tokamaks where the mode activity is usually concentrated on the outer side. The characteristic poloidal scale of the mode changes with the poloidal angle. In Fig. 11, the modification of the electrostatic potential structure with the toroidal angle is shown at the flux surface $s=0.5$. One sees that the mode has a flute-like character (one 
stellarator period is shown here).

Now, consider the effect of the density profile. In Fig. 12, the frequency and the growth rate are plotted as functions of the inverse characteristic length of the density profile, see Eq. (25). The frequency $\omega$ and the growth rate $\gamma$ of the mode is determined fitting the function $f(t)=\exp (\gamma t)(a \cos \omega t+b \sin \omega t)$ to a signal obtained in the simulations, such as plotted in Fig. 7. The accuracy of such a fit is usually within few percents. Interestingly, the growth rate is nearly insensitive with respect to the density profile in the electromagnetic case, in contrast to the usual ITG mode which can be stabilised at a larger density gradient or smaller $\eta_{\mathrm{i}}$. We hypothesise that the ITG stabilisation by the Alfvén wave coupling is compensated by some drive, caused for example by the ballooning or the trapped-electron effects. Much more simulations will be needed in future to address this physics in more detail. The purpose of this paper is to demonstrate a principle feasibility of such simulations.

The mode remains slab-like also at a finite density gradient, as shown in Fig. 13, where the Fourier decomposition of the mode is plotted measured at the flux surface $s=0.5$. Here, the inverse characteristic length of the density profile $1 / L_{\mathrm{n}}=3.0$. The poloidal cross-section of the electrostatic potential at the toroidal angle $\zeta=0.0$ for the case $1 / L_{\mathrm{n}}=3.0$ is plotted in Fig. 14. One sees that here, similarly to Fig. 10, the mode develops both on the inner and the outer sides of the torus. Again, the characteristic poloidal scale of the mode changes with the poloidal angle.

\section{CONCLUSIONS}

In this paper, we have presented the GYGLES/EUTERPE toolset developed to study global electromagnetic modes in tokamak and stellarator geometries. A fully gyrokinetic model, both in the standard and in the mixed-variable formulations, and two reduced models, the fluid-electron and the perturbative hybrid MHD model, have been formulated.

The mixed-variable formulation makes electromagnetic global fully gyrokinetic simulations in complicated shaped magnetic geometries feasible. In this paper, we have presented TAE simulations in elongated tokamak geometry and electromagnetic ITG simulations in W7-X stellarator geometry.

In the tokamak, the elongation effect on the frequency and the growth rate has been studied. The frequency is modified according to continuum reshaping caused by new gaps 
which are created by the elongation. In our simulations, the growth rate is decreased and the mode stabilised by the plasma elongation, similarly to the finding of Ref. [22]. There it was suggested that the reduction of mode growth rates due to elongation is mainly the result of reduced fast-particle drive. Elongation in effect increases the average radius and reduces the average radial gradient of the fast-particle distribution.

In the stellarator, we have considered the ITG instability including electromagnetic effects. It has been found that this mode has a slab-like character and is not stabilised by the finite density gradient, in contrast to the conventional ITG intuition which suggests that the mode is stable at small $\eta_{\mathrm{i}}=\nabla \log T_{\mathrm{i}} / \nabla \log n$. In fact, the growth rate of the electromagnetic mode is only weakly affected by the density gradient and the mode structure remains slab-like. This result is of practical interest for W7-X where the conventional electrostatic ITG mode could be stabilised by a finite density gradient. In this paper, we have shown that such a stabilisation will not necessarily be achieved when the electromagnetic effects are taken into account. As a word of caution, we note that benchmarks with other codes and models are needed and forseen in order to establish the reliability of the code and its results in stellarator geometry. This work has already been started in Ref. [24] and will be continued in future. In tokamak geometry, benchmarks beyond the ITPA case [20] have been performed in the present paper for the elongated tokamak geometry. Further benchmarks have been described in Ref. [24].

We plan to expand our stellarator studies towards effects caused by radial electric fields, collisions and nonlinearity. Both electromagnetic microturbulence and the Alfvénic eigenmodes, such as the stellarator TAE, will be considered in this research. The fully gyrokinetic treatment of stellarator plasmas allows for a consistent description of damping mechanisms which were lacking in our previous study [17]. The reduced modelling will be expanded to include resistive effects and fluid-electron nonlinearities.

\section{ACKNOWLEDGMENTS}

We acknowledge P. Helander for his support. We thank Jürgen Nührenberg for reading and commenting our manuscript. We appreciate comments of the unknown referees helping us to improve the paper. This work was carried out using the HELIOS supercomputer system at Computational Simulation Centre of International Fusion Energy Research Centre 
(IFERC-CSC), Aomori, Japan, under the Broader Approach collaboration between Euratom and Japan, implemented by Fusion for Energy and JAEA. Also, some simulations have been performed on the local cluster in Greifswald, where support of Henry Leyh is appreciated. This project has received funding from the European Union's Horizon 2020 research and innovation programme under grant agreement number 633053. The views and opinions expressed herein do not necessarily reflect those of the European Commission.

[1] Y. Chen and S. Parker, Phys. Plasmas 8, 2095 (2001).

[2] A. Mishchenko, R. Hatzky, and A. Könies, Phys. Plasmas 11, 5480 (2004).

[3] Y. Chen and S. Parker, J. Comp. Phys 189, 463 (2003).

[4] A. Mishchenko, A. Könies, and R. Hatzky, in Proc. of the Joint Varenna-Lausanne International Workshop (Società Italiana di Fisica, Bologna, 2004).

[5] R. Hatzky, A. Könies, and A. Mishchenko, J. Comp. Phys. 225, 568 (2007).

[6] J. Candy and R. E. Waltz, J. Comp. Phys. 186, 545 (2003).

[7] A. Mishchenko, M. Cole, R. Kleiber, and A. Könies, Phys. Plasmas 21, 052113 (2014).

[8] A. Mishchenko, A. Könies, R. Kleiber, and M. Cole, Phys. Plasmas 21, 092110 (2014).

[9] W. Lotz, J. Nührenberg, and C. Schwab, in Proceedings of the 13th International Conference on Plasma Physics and Controlled Nuclear Fusion Research (Washington, DC, 1990) (International Atomic Energy Agency, Vienna, 1991), Vol. 3, p. 603.

[10] A. Mishchenko, R. Hatzky, and A. Könies, Phys. Plasmas 15, 112106 (2008).

[11] A. Mishchenko, A. Könies, and R. Hatzky, Phys. Plasmas 16, 082105 (2009).

[12] A. Mishchenko and A. Könies and R. Hatzky, Phys. Plasmas 18, 012504 (2011).

[13] A. Mishchenko and A. Zocco, Phys. Plasmas 19, 122104 (2012).

[14] A. Mishchenko, A. Könies, and R. Hatzky, Phys. Plasmas 21, 052114 (2014).

[15] S. P. Hirshman, W. I. van Rij, and P. Merkel, Comp. Phys. Commun. 43, 143 (1986).

[16] T. Fehér, Ph.D. thesis, Max-Planck-Institut für Plasmaphysik, Greifswald, 2013.

[17] A. Mishchenko, A. Könies, T. Fehér, R. Kleiber, M. Borchardt, J. Riemann, R. Hatzky, J. Geiger, and Yu. Turkin, Nucl. Fusion 54, 104003 (2014).

[18] A. Könies, in IAEA TM on Energetic Particles (IAEA, Kloster Seon, 2007).

[19] M. Cole, A. Mishchenko, A. Könies, R. Kleiber, and M. Borchardt, Phys. Plasmas 21, 072123 
(2014).

[20] A. Könies, S. Briguglio, N. Gorelenkov, T. Fehér, M. Isaev, P. Lauber, A. Mishchenko, D. A. Spong, Y. Todo, W. A. Cooper, R. Hatzky, R. Kleiber, M. Borchardt, G. Vlad, and ITPA EP TG, "Benchmark of gyrokinetic, kinetic MHD and gyrofluid codes for the linear calculation of fast particle driven TAE dynamics", paper presented at 24th IAEA Int. Conf. on Fusion Energy, San Diego 2012).

[21] A. J. Brizard and T. S. Hahm, Reviews of Modern Physics 79, 421 (2007).

[22] Y. Chen, S. Parker, J. Lang, and G.-Y. Fu, Phys. Plasmas 17, 102504 (2010).

[23] G. G. Plunk, P. Helander, P. Xanthopoulos, and J. W. Connor, Phys. Plasmas 21, 032112 (2014).

[24] M. Cole, A. Mishchenko, A. Könies, R. Hatzky, and R. Kleiber, accepted in Plasma Phys. Controlled Fusion (2015). 


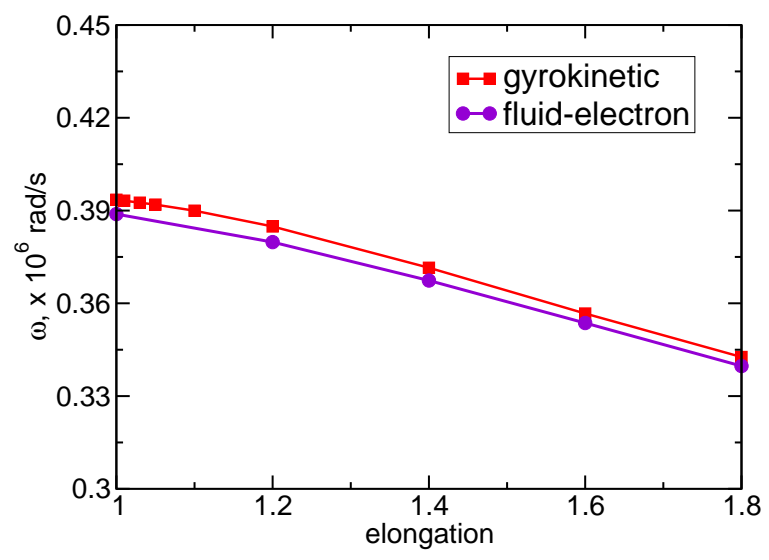

FIG. 1: (Color online) The frequency of the TAE mode as a function of the elongation. Tokamak geometry is considered here. The gyrokinetic simulations are compared with the fluid-electron model and a good agreement is found. 


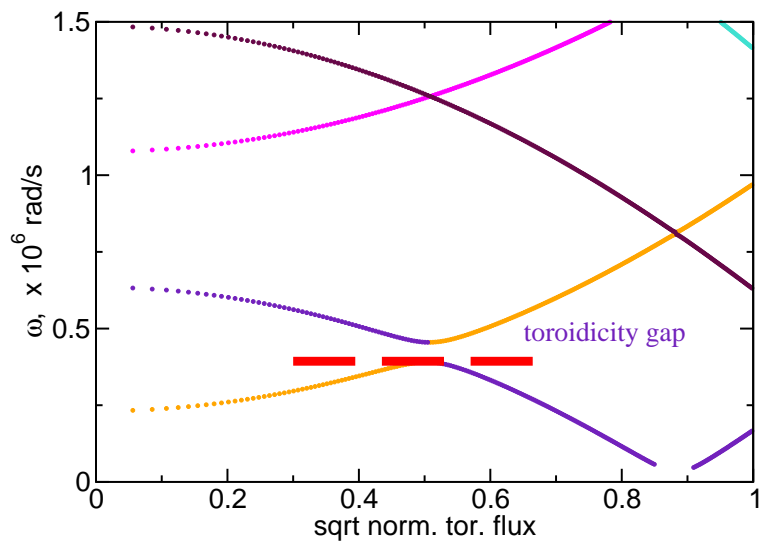

FIG. 2: (Color online) Shear Alfvén continuum at the elongation $\kappa=1$. The toroidicity gap is observed. Tokamak geometry is considered here. The TAE frequency corresponding to the elongation $\kappa=1$ is indicated with the red dashed line. 


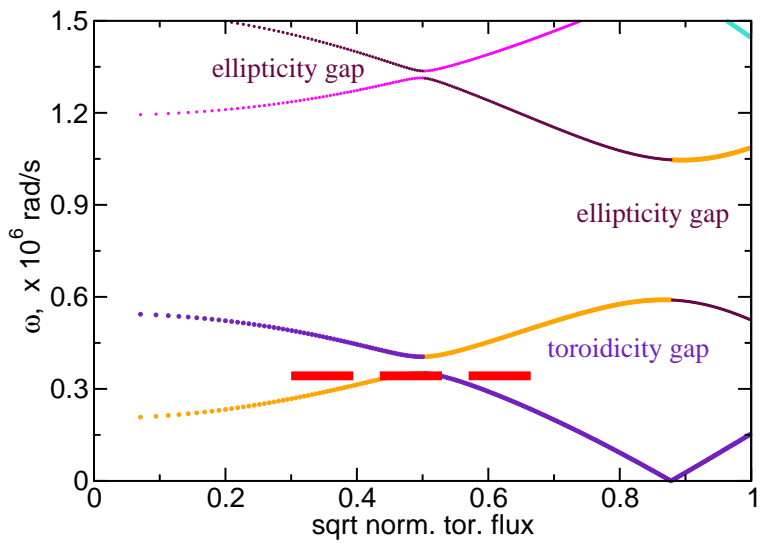

FIG. 3: (Color online) Shear Alfvén continuum at the elongation $\kappa=1.8$. The toroidicity and the ellipticity gaps are observed. The TAE eigenmode frequency is indicated with the red dashed line. One sees that the elliptic coupling of the poloidal Fourier harmonics pushes the toroidal gap to the lower frequencies. Tokamak geometry is considered here. 


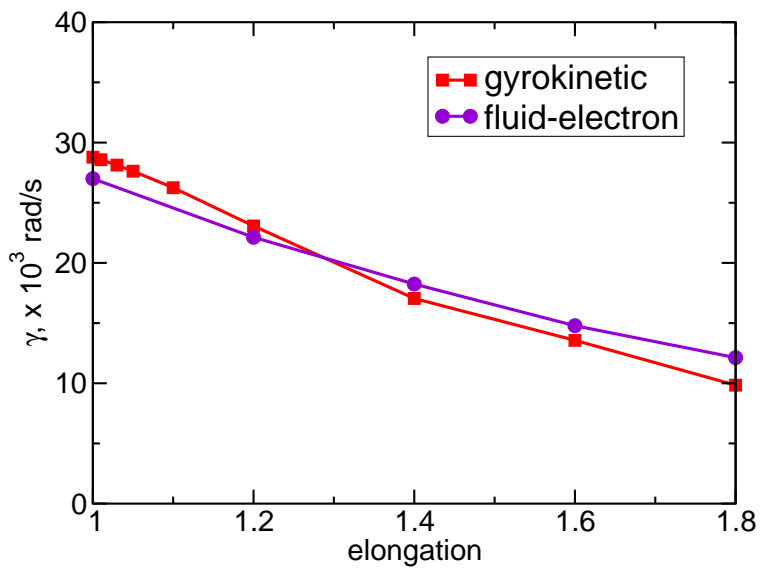

FIG. 4: (Color online) The growth rate of the TAE mode as a function of the elongation. Tokamak geometry is considered here. The gyrokinetic simulations are compared with the fluid-electron model and a good agreement is found. 


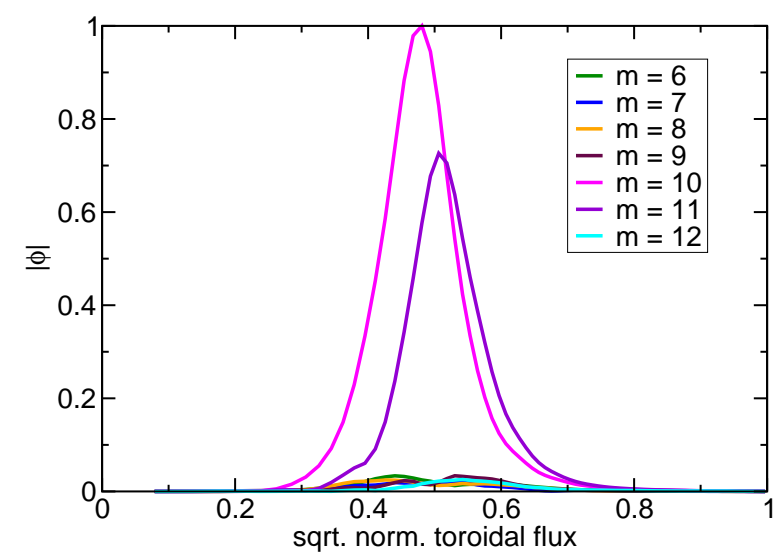

FIG. 5: (Color online) Eigenmode structure of the electrostatic potential computed at the elongation $\kappa=1.2$. A typical TAE structure can be seen at this elongation. Tokamak geometry is considered here. 


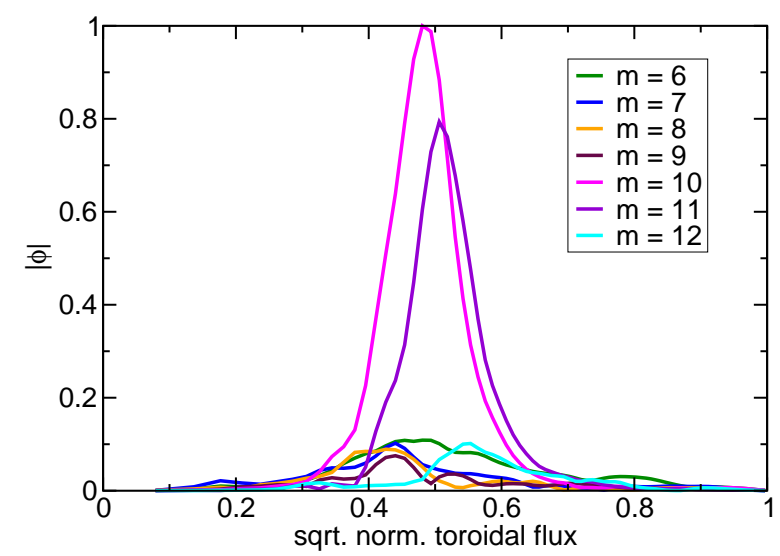

FIG. 6: (Color online) Eigenmode structure of the electrostatic potential computed at the elongation $\kappa=1.8$. The coupling to the poloidal sidebands is stronger here compared to Fig. 5. Tokamak geometry is considered on this plot. 


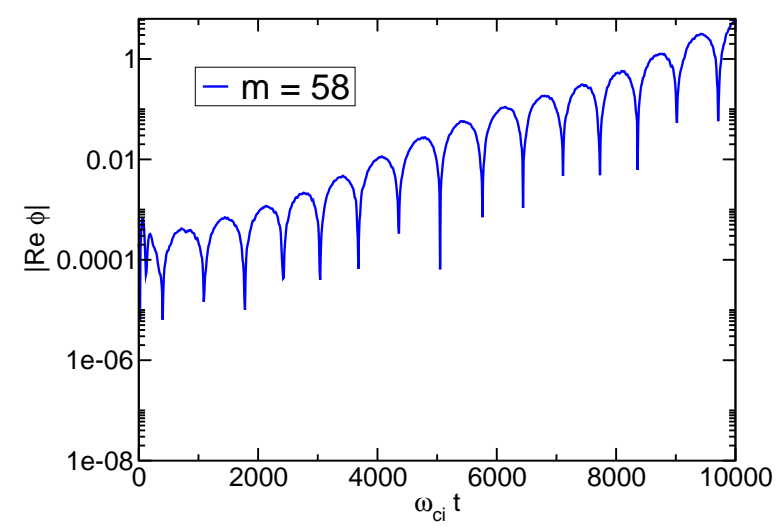

FIG. 7: (Color online) Temporal evolution of the dominant $m=58$ poloidal Fourier component of the electrostatic potential $\phi(s=0.56, \theta, \zeta=0)$. An electromagnetic ITG mode is considered in W7-X for $\beta_{*}=0.005$ (which corresponds to a characteristic physical $\beta=2 \%$ ). 


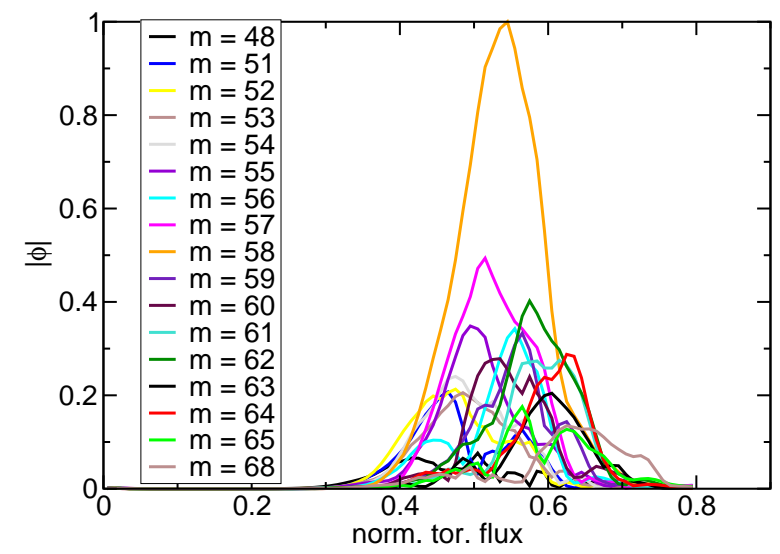

FIG. 8: (Color online) Poloidal Fourier decomposition of the electrostatic potential $\phi(s, \theta, \zeta=0)$. W7-X geometry is considered here. 


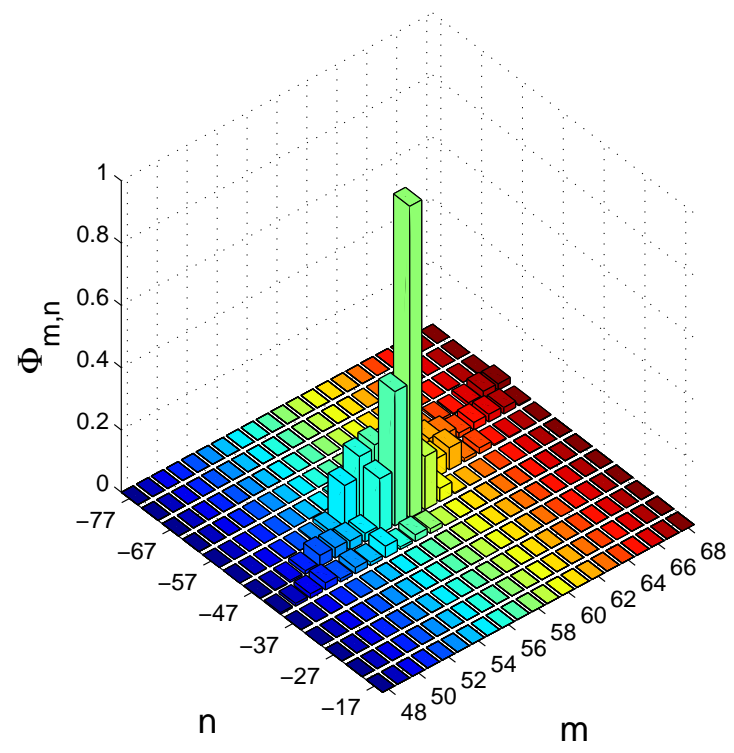

FIG. 9: (Color online) Fourier spectrum measured at $s=0.5$. Here, the density profile is flat. The spectrum has a dominant harmonic (slab-like). W7-X geometry is considered on this plot. 


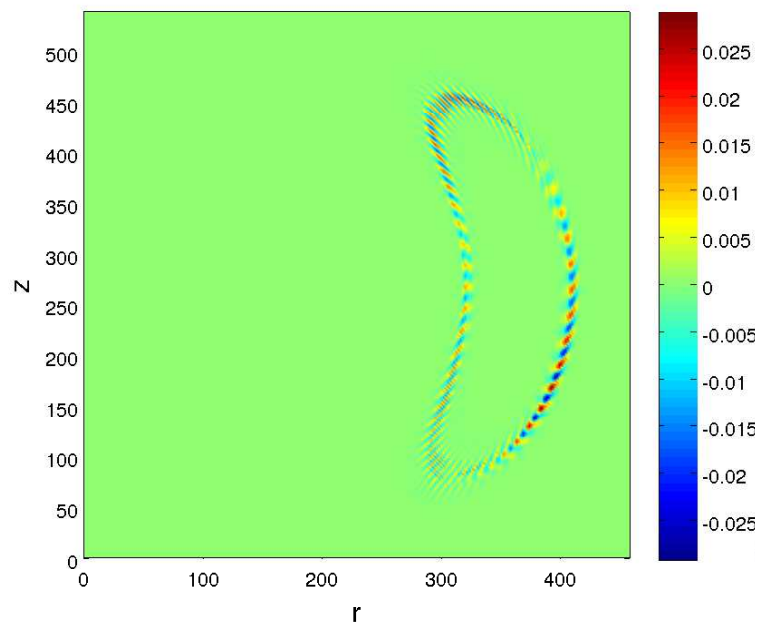

FIG. 10: (Color online) Poloidal cross-section of the electrostatic potential measured at $\zeta=0$ for a flat density profile. It is observed that the characteristic poloidal scale of the mode changes with the poloidal angle. W7-X geometry is considered here. 


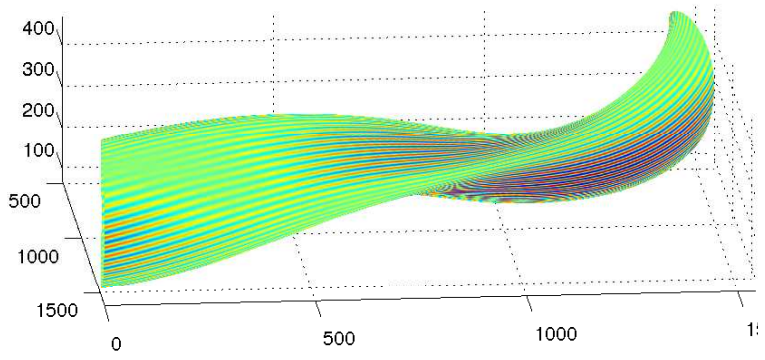

FIG. 11: (Color online) Modification of the electrostatic potential structure with the toroidal angle measured at $s=0.5$ (one stellarator period is shown). W7-X geometry is considered here. 


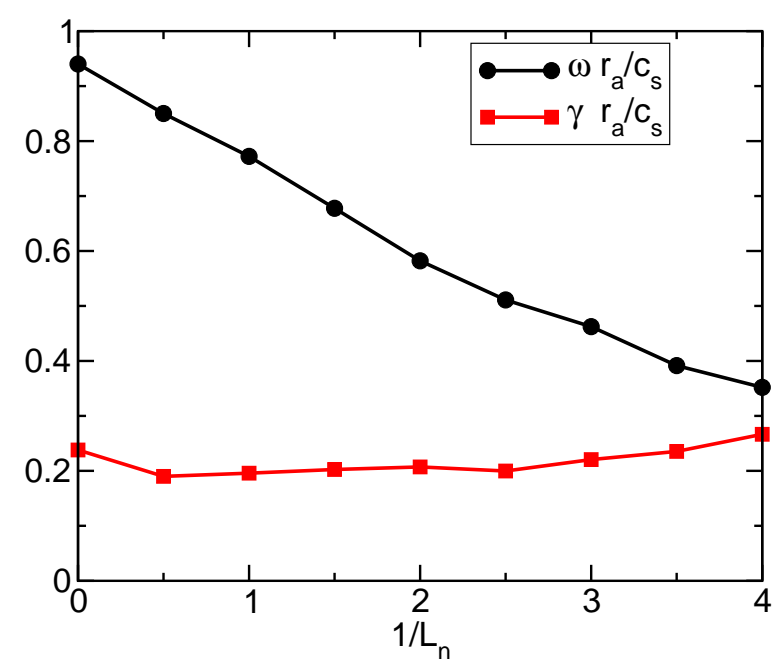

FIG. 12: (Color online) The frequency and the growth rate as functions of the density gradient. The normalisation to $2.2 \omega_{\mathrm{ci}} / L_{x} \approx c_{\mathrm{s}} / r_{\mathrm{a}}$ is used. The ion temperature gradient $1 / L_{\mathrm{Ti}}=3.5$ and a flat electron temperature profile have been used. Interestingly, the growth rate is nearly insensitive with respect to the density profile, in contrast to the usual ITG behaviour. W7-X geometry is considered here. 


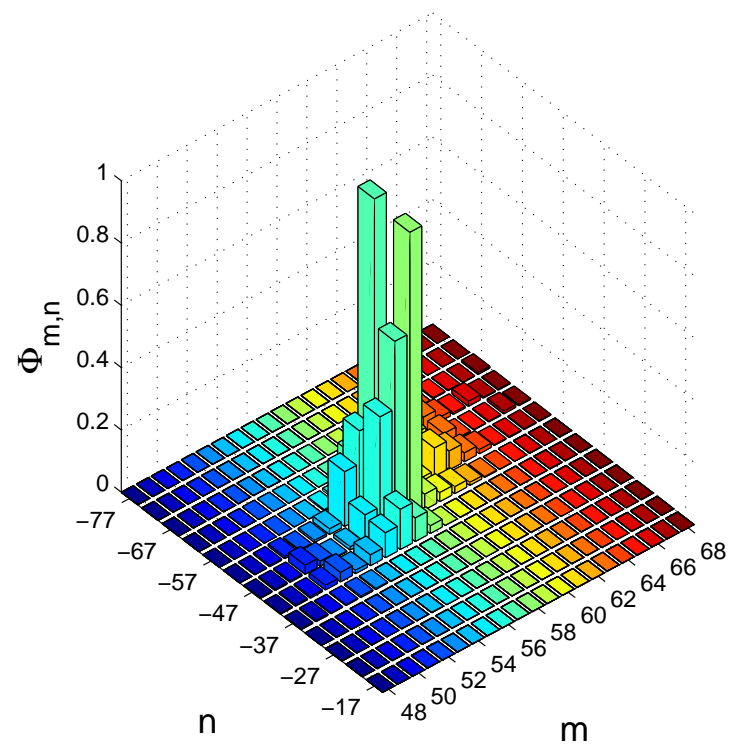

FIG. 13: (Color online) Fourier spectrum for the EM mode at $\kappa_{\mathrm{n}}=3.0$ (finite density gradient). The electromagnetic Fourier spectrum remains slab-like also at the finite density gradient. W7-X geometry is considered here. 


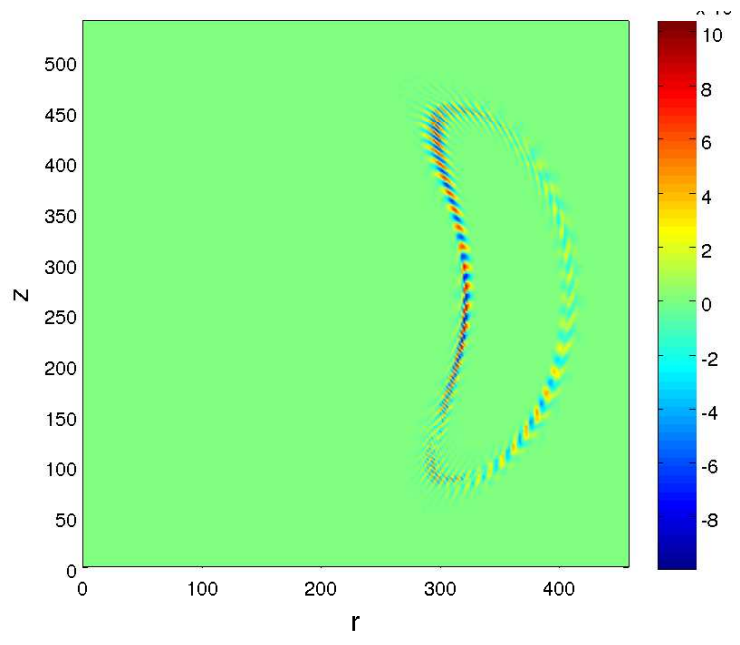

FIG. 14: (Color online) Poloidal cross-section of the electrostatic potential of an electromagnetic ITG mode in W7-X measured at the toroidal angle $\zeta=0$ for the inverse characteristic length of the density profile $1 / L_{\mathrm{n}}=3.0$. Note the change in the characteristic poloidal scale of the mode with the poloidal angle. 